\title{
$\mathrm{O}_{1}$-convergence in partially ordered sets
}

\author{
Tao Sun ${ }^{\mathrm{a}, \mathrm{b}}$, Qingguo Lij,*, Nianbai Fan ${ }^{\mathrm{c}}$ \\ ${ }^{a}$ College of Mathematics and Physics, Hunan University of Arts and Science, Changde, Hunan 415000, P. R. China. \\ ${ }^{b}$ College of Mathematics and Econometrics, Hunan University, Changsha, Hunan 410082, P. R. China. \\ ${ }^{c}$ College of Computer Science and Electronic Engineering, Hunan University, Changsha, Hunan 410082, P. R. China.
}

\begin{abstract}
Based on the introduction of notions of $S^{*}$-doubly continuous posets and B-topology in [T. Sun, Q. G. Li, L. K. Guo, Topology Appl., 207 (2016), 156-166], in this paper, we further propose the concept of B-consistent $S^{*}$-doubly continuous posets and prove that the $\mathrm{O}_{1}$-convergence in a poset is topological if and only if the poset is a $\mathrm{B}$-consistent $\mathrm{S}^{*}$-doubly continuous poset. This is the main result which can be seen as a sufficient and necessary condition for the $\mathrm{O}_{1}$-convergence in a poset being topological. Additionally, in order to present natural examples of posets which satisfy such condition, several special sub-classes of B-consistent $\mathrm{S}^{*}$-doubly continuous posets are investigated.
\end{abstract}

Keywords: $\mathrm{O}_{1}$-convergence, B-topology, $\mathrm{S}^{*}$-doubly continuous poset, B-consistent $\mathrm{S}^{*}$-doubly continuous poset.

2010 MSC: 54A20, 06A06.

(C)2019 All rights reserved.

\section{Introduction and preliminaries}

The concept of O-convergence in partially ordered sets (posets, for short) was introduced by Birkhoff [1], Frink [5], and Mcshane [9]. It is defined as follows: a net $\left(x_{i}\right)_{i \in I}$ in a poset $P$ is said to O-converges to $x \in \mathrm{P}$ (we write $\left(x_{i}\right)_{i \in \mathrm{I}} \stackrel{\mathrm{O}}{\rightarrow} \mathrm{x}$ in this paper) if there exist subsets $\mathrm{D}$ and $\mathrm{F}$ of $\mathrm{P}$ such that

(1) $\mathrm{D}$ is directed and $\mathrm{F}$ is filtered;

(2) $\sup D=x=\inf F$;

(3) for every $d \in D$ and $e \in F, d \leqslant x_{i} \leqslant e$ holds eventually, i.e., there exists $i_{0} \in$ I such that $d \leqslant x_{i} \leqslant e$ for all $i \geqslant i_{0}$.

As what has been showed in [18], the O-convergence (Note: in [18], the O-convergence is called orderconvergence) in a general poset $\mathrm{P}$ may not be topological, i.e., it is possible that $\mathrm{P}$ can not be endowed with a topology such that the O-convergence and the associated topological convergence are consistent. Hence, much works has been done to characterize those special posets in which the O-convergence is topological. The most recent result in [13] shows that the O-convergence in a poset which satisfies condition $(\triangle)$ is

\footnotetext{
*Corresponding author

Email addresses: suntao5771@163.com (Tao Sun), liqingguoli@aliyun.com (Qingguo Li), nbfan6203@163.com (Nianbai Fan)

doi: $10.22436 /$ jnsa.012.10.02
}

Received: 2017-04-03 Revised: 2019-02-27 Accepted: 2019-04-30 
topological if and only if the poset is $\mathcal{O}$-doubly continuous. This means that for a special class of posets, a sufficient and necessary condition for $\mathrm{O}$-convergence being topological is obtained. For more results on O-convergence, the reader can refer to $[8,10,14,17]$.

The $\mathrm{O}_{1}$-convergence is a special type of $\mathrm{O}$-convergence in posets and was also introduced by Birkhoff [1]. In fact, the $\mathrm{O}_{1}$-convergence in a general poset may also not be topological. To search those special posets in which the $\mathrm{O}_{1}$-convergence is topological, Riecanová [11] proved that the $\mathrm{O}_{1}$-convergence in any separable strongly compactly atomistic orthomodular lattice is topological. This result clarified a special condition of posets under which the $\mathrm{O}_{1}$-convergence is topological. However, to the best of our knowledge, the equivalent characterization to the $\mathrm{O}_{1}$-convergence in general posets being topological is still unknown.

We continue to consider the $\mathrm{O}_{1}$-convergence in poset with the aim of establishing the equivalent characterization to the $\mathrm{O}_{1}$-convergence in general posets being topological. More specifically, given a general poset $\mathrm{P}$, we hope to clarify the order-theoretical condition of $\mathrm{P}$ which is sufficient and necessary for the associated $\mathrm{O}_{1}$-convergence being topological. To this end, in Section 2, we propose the notion of B-consistent $S^{*}$-doubly continuous posets by introducing condition $(\star)$, and then obtain the main result of this paper, that is: given a poset $\mathrm{P}$, the $\mathrm{O}_{1}$-convergence in $\mathrm{P}$ is topological if and only if $\mathrm{P}$ is a $\mathrm{B}$ consistent $\mathrm{S}^{*}$-doubly continuous poset if and only if the $\mathrm{O}_{1}$-convergence and the topological convergence with respect to the B-topology on $\mathrm{P}$ are consistent. In Section 3, we study several special sub-classes of B-consistent $\mathrm{S}^{*}$-doubly continuous posets.

Some conventional notions will be used in the sequel. Throughout this paper, given a set $X, F \sqsubseteq X$ means that $F$ is a finite subset of $X$. Let $P$ be a poset and $x \in P, \uparrow x$ and $\downarrow x$ are always used to denote the principal filter $\{y \in P: y \geqslant x\}$ and the principal ideal $\{z \in P: z \leqslant x\}$ of $P$, respectively. $\mathrm{P}$ is said to be bounded if it has the least element $\perp$ and the largest element $T$. Given a poset $P$ and $A \subseteq P$, by writing sup $A$ we mean that the least upper bound of $A$ in $P$ exists and equals to sup $A \in P$; dually, by writing inf $A$ we mean that the greatest lower bound of $A$ in $P$ exists and equals to $\inf A \in P$. And the set $A$ is called an upper set if $A=\uparrow A=\{b \in P:(\exists a \in A) a \leqslant b\}$, the lower set is defined dually.

Given a topological space $(X, \mathcal{T})[4,7]$ and a net $\left(x_{i}\right)_{i \in I}$ in $X$, we take $\left(x_{i}\right)_{i \in I} \stackrel{\mathcal{T}}{\rightarrow} x \in X$ to mean that the net $\left(x_{i}\right)_{i \in I}$ converges to $x$ with respect to the topology $\mathcal{T}$.

To make this paper self-contained, we briefly review the following notions and propositions.

Definition 1.1 ([6]). Let $P$ be a poset and $x, y, z \in P$. We say $y \ll x$ if for every directed subset $D$ of $P$ with $x \leqslant \sup D$, there exists $d \in D$ such that $y \leqslant d$; dually, we say $z \triangleright x$ if for every filtered subset $F$ of $P$ with inf $F \leqslant x$, there exists $e \in F$ such that $e \leqslant z$.

Remark 1.2 ([6]). Let $\mathrm{P}$ be a poset and $x, y, z, a, b, c, d \in P$. Then

(1) $x \ll y \Rightarrow x \leqslant y$ and $z \triangleright x \Rightarrow z \geqslant x$;

(2) $a \leqslant x \ll y \leqslant b \Rightarrow a \ll b$ and $c \geqslant z \triangleright x \geqslant d \Rightarrow c \triangleright d$.

Definition 1.3 ([16]). A poset $P$ is called a doubly continuous poset if for any $x \in P$, the set $\{y \in P: y \ll x\}$ is directed, the set $\{z \in P: z \triangleright x\}$ is filtered and $\sup \{y \in P: y \ll x\}=x=\inf \{z \in P: z \triangleright x\}$.

Remark 1.4 ([15]). Let $P$ be a doubly continuous poset and $x, y, z \in P$. If $x \ll y$, then there exists $a \in P$ such that $x \ll a \ll y$; dually, if $z \triangleright x$, then there exists $b \in P$ such that $z \triangleright b \triangleright x$.

Example 1.5 ([3, 19]).

(1) Chains, antichains, and finite posets are all doubly continuous posets.

(2) Every completely distributive lattice is a doubly continuous lattice. But the converse may not be true. For example, every non-distributive finite lattice is a doubly continuous lattice, but not a completely distributive lattice. In fact, it has been shown that $\mathrm{L}$ is a completely distributive lattice if and only if it is a distributive doubly continuous lattice. 
Definition 1.6 ([12]). Let $P$ be a poset and $x, y, z \in P$. We define $y \ll s x$ if for every directed subset $D$ of $P$ with $\sup D=x$, there exists $d \in D$ such that $y \leqslant d$; dually, we define $z \triangleright s x$ if for every filtered subset $F$ of $P$ with $\inf F=x$, there exists $e \in F$ such that $z \geqslant e$.

In what follows, for a poset $\mathrm{P}$ and $x \in \mathrm{P}$, we denote

(1) $\Downarrow_{S} x=\{a \in P: a \ll s x\}, \Uparrow s x=\{b \in P: x \ll s b\} ;$

(2) $\Downarrow_{s} x=\{c \in P: x \triangleright s c\}, \uparrow_{s} x=\{d \in P: d \triangleright s x\}$.

Definition 1.7 ([12]). A poset $P$ is said to be S-doubly continuous if for every $x \in P$, the sets $\Downarrow_{s} \chi$ and $\uparrow_{s} x$ are directed and filtered, respectively, and $\sup \Downarrow_{s} x=x=\inf \uparrow_{s} x$.

Definition 1.8 ([12]). An $S$-doubly continuous poset $P$ is said to be $S^{*}$-doubly continuous if for every $x \in P, y \in \Downarrow_{S} x$ and $z \in \uparrow_{s} x$, there exist $y_{0} \in \Downarrow_{s} x$ and $z_{0} \in \uparrow_{s} x$ such that $\uparrow_{0} \cap \downarrow_{z_{0}} \subseteq \Uparrow s y \cap \downarrow_{s} z$.

Proposition 1.9 ([12]). If $\mathrm{P}$ is a doubly continuous poset, then $\mathrm{P}$ is $\mathrm{S}^{*}$-doubly continuous.

Definition 1.10 ([6]). Let $\mathrm{P}$ be a poset and $\mathrm{U} \subseteq \mathrm{P}$. $\mathrm{U}$ is said to be Scott open if and only if the the following two conditions are satisfied:

(1) $\mathrm{U}=\uparrow \mathrm{U}$, that is to say, $\mathrm{U}$ is an upper set;

(2) $\sup D \in U$ implies $D \cap U \neq \emptyset$ for every directed subset $D$ of $P$.

It can be formally verified that the collection of all Scott open subsets of P forms a topology on P, which is called the Scott topology and denoted by $\sigma_{\mathrm{P}}$.

\section{B-consistent $S^{*}$-doubly continuous posets}

In this section, the $\mathrm{O}_{1}$-convergence in posets is reviewed. Then, a special class of $\mathrm{S}^{*}$-doubly continuous posets, named B-consistent $S^{*}$-doubly continuous posets, is introduced. Finally, we present a sufficient and necessary condition of a general poset which can precisely serve as an order-theoretical condition for the associated $\mathrm{O}_{1}$-convergence being topological.

Definition 2.1 ([1]). Let $P$ be a poset. A net $\left(x_{i}\right)_{i \in I}$ in $P$ is said to $O_{1}$-converges to $x \in P$ if there exist nets $\left(u_{i}\right)_{i \in I}$ and $\left(v_{i}\right)_{i \in I}$ in $P$ such that

(O1) $\left(u_{i}\right)_{i \in I}$ is an increasing net, i.e., $u_{i_{1}} \leqslant u_{i_{2}}$ for any $i_{1}, i_{2} \in I$ with $i_{1} \leqslant i_{2}$, and $\left(v_{i}\right)_{i \in I}$ is a decreasing net, i.e., $v_{i_{1}} \geqslant v_{i_{2}}$ for any $i_{1}, i_{2} \in I$ with $i_{1} \leqslant i_{2}$

(O2) $u_{i} \leqslant x_{i} \leqslant v_{i}$ for all $i \in I$;

(O3) $\sup \left\{u_{i}: i \in I\right\}=x=\inf \left\{v_{i}: i \in I\right\}$.

In this case, we write $\left(x_{i}\right)_{i \in I} \stackrel{\mathrm{O}_{1}}{\longrightarrow} x$.

It is worth noting that if $\left(x_{i}\right)_{i \in I}$ is a net in a poset $P$, then $\left(x_{i}\right)_{i \in I} \stackrel{\mathrm{O}_{1}}{\rightarrow} x \in P$ implies $\left(x_{i}\right)_{i \in I} \stackrel{O}{\rightarrow} x$. But the converse implication may not be true. This fact can be illustrated by the following Example 2.2.

Example 2.2. Let $P=\{a, b, x\}$ with $a \leqslant x$ and $b \leqslant x$, and $I=\{1,2,3\}$ with $1 \leqslant 2 \leqslant 3$. And let $\left(x_{i}\right)_{i \in I}$ be the net defined by $x_{1}=a, x_{2}=b$ and $x_{3}=x$. By the definition of O-convergence, it is easy to verify that $\left(x_{i}\right)_{i \in I} \stackrel{O}{\rightarrow} x$. Suppose $\left(x_{i}\right)_{i \in I} \stackrel{\mathrm{O}_{1}}{\rightarrow} x$. Then there exist an increasing net $\left(u_{i}\right)_{i \in I}$ and a decreasing net $\left(v_{i}\right)_{i \in I}$ which satisfy the conditions (O2) and (O3) in Definition 2.1. By (O2), we have $u_{1} \leqslant x_{1}=a \leqslant v_{1}$ and $u_{2} \leqslant x_{2}=b \leqslant v_{2}$, which implies that $u_{1}=a, u_{2}=b$ and $u_{1} \nless u_{2}$. This contradicts the fact that $\left(u_{i}\right)_{i \in I}$ is an increasing net. Thus, the net $\left(x_{i}\right)_{i \in I}$ does not $\mathrm{O}_{1}$-converge to $x$.

Remark 2.3. Let $\mathrm{P}$ be a poset and $\left(\mathrm{x}_{\mathfrak{i}}\right)_{i \in \mathrm{I}}$ a net in $\mathrm{P}$. 
(1) If $\left(x_{i}\right)_{i \in I}$ is a constant net in $P$ with value $x$, then $\left(x_{i}\right)_{i \in I} \stackrel{O_{1}}{\longrightarrow} x$ since the increasing net $\left(u_{i}\right)_{i \in I}$ defined by $u_{i}=x$ for all $i \in I$ and the decreasing net $\left(v_{i}\right)_{i \in I}$ defined by $v_{i}=x$ for all $i \in$ I satisfy the conditions (O2) and (O3) in Definition 2.1.

(2) Suppose $\left(x_{i}\right)_{i \in I} \stackrel{\mathrm{O}_{1}}{\longrightarrow} x \in P$. Then there exist an increasing net $\left(u_{i}\right)_{i \in I}$ and a decreasing net $\left(v_{i}\right)_{i \in I}$ in $P$ that satisfy the conditions (O2) and (O3) in Definition 2.1. This can imply that the subsets $\left\{u_{i}: i \in I\right\}$ and $\left\{v_{i}: i \in I\right\}$ of $P$ are directed and filtered, respectively.

(3) The $\mathrm{O}_{1}$-convergent point of a net $\left(x_{i}\right)_{i \in I}$ in $P$, if exists, is unique. Indeed, suppose $\left(x_{i}\right)_{i \in I} \stackrel{\mathrm{O}_{1}}{\longrightarrow} x_{1} \in P$ and $\left(x_{i}\right)_{i \in I} \stackrel{\mathrm{O}_{1}}{\longrightarrow} x_{2} \in P$. Then we have $\left(x_{i}\right)_{i \in I} \stackrel{\mathrm{O}}{\rightarrow} x_{1}$ and $\left(x_{i}\right)_{i \in I} \stackrel{\mathrm{O}}{\rightarrow} x_{2}$. By Remark 2.3 (2) (the O-convergent point of a net $\left(x_{i}\right)_{i \in I}$ in $P$, if exists, is unique.) in [19], $x_{1}=x_{2}$.

(4) Let $D$ be a directed subset of $P$ and $F$ a filtered subset of $P$ such that sup $D$ and inf F exist. Define the net $\left(x_{d}\right)_{d \in D}$ by $x_{d}=d$ for all $d \in D$ and the net $\left(y_{e}\right)_{e \in F o p}$ by $y_{e}=e$ for all $e \in F$. Then $\left(x_{d}\right)_{d \in D} \stackrel{O_{1}}{\longrightarrow} \sup D$ and $\left(y_{e}\right)_{e \in F o p} \stackrel{\mathrm{O}_{1}}{\longrightarrow} \inf F$.

Next we recall the definition and the fundamental properties of B-topology on posets, which has been introduced in [12].

Definition 2.4 ([12]). Given a poset $\mathrm{P}$, a subset $\mathrm{U} \subseteq \mathrm{P}$ is called a $B$-open set if for any filter $\mathcal{F}$ in $\mathrm{P}$ that orderconverges to $x \in \mathrm{U}$, there exists $\mathrm{F} \in \mathcal{F}$ such that $\mathrm{F} \subseteq \mathrm{U}$. (Note: for the definition of order-convergence in posets, one can refer to Definition 2.1 in [12].)

For a poset $P$, let $\mathcal{T}_{P}$ denote the set of all B-open subsets of $P$. It is routine to check that $\mathcal{T}_{P}$ forms a topology on P. And this topology is called the B-Topology on P.

Proposition 2.5 ([12]). Let $\mathrm{P}$ be a poset and $\mathrm{U} \subseteq \mathrm{P}$. Then $\mathrm{U} \in \mathcal{T}_{\mathrm{P}}$ if and only if for any directed subset $\mathrm{D}$ of $\mathrm{P}$ and any filtered subset $\mathrm{F}$ of $\mathrm{P}$ with $\sup \mathrm{D}=\inf \mathrm{F}=\mathrm{x} \in \mathrm{U}$, there exist $\mathrm{d}_{0} \in \mathrm{D}$ and $\mathrm{e}_{0} \in \mathrm{F}$ such that $\uparrow \mathrm{d}_{0} \cap \downarrow e_{0} \subseteq \mathrm{U}$.

Recall that given a topological space $(X, \mathcal{T})$, a subfamily $\mathcal{B}$ of $\mathcal{T}$ is called an open base for the topological space $(X, \mathcal{T})$ (sometimes, called an open base of $\mathcal{T}$ ) if $\mathcal{B} \subseteq \mathcal{T}$ and for every point $x \in X$ and every neighborhood $\mathrm{V}$ of $\mathrm{x}$ there exists $\mathrm{U} \in \mathcal{B}$ such that $\mathrm{x} \in \mathrm{U} \subseteq \mathrm{V}$.

Theorem 2.6 ([12]). If $\mathrm{P}$ is $\mathrm{S}^{*}$-doubly continuous, then $\mathcal{B}_{\mathrm{P}}=\left\{\Uparrow_{\mathrm{S}} \mathrm{y} \cap \downarrow_{\mathrm{S}} z: y, z \in \mathrm{P}\right\}$ forms an open base of the B-topology $\mathcal{T}_{\mathrm{P}}$.

Definition 2.7 ([16]). Let $\mathrm{P}$ be a poset. A family $\bar{\sigma}_{\mathrm{P}}$ of subsets of $\mathrm{P}$ is called the Bi-Scott topology on $\mathrm{P}$ if $\bar{\sigma}_{\mathrm{P}}=$ $\sigma_{\mathrm{P}} \vee \sigma_{\mathrm{POP}}$, where $\mathrm{P}^{\mathrm{OP}}$ is the dual poset of $\mathrm{P}$. Obviously, $\bar{\sigma}_{\mathrm{P}}$ has an open base $\mathcal{B}=\left\{\mathrm{U} \cap \mathrm{V}: \mathrm{U} \in \sigma_{\mathrm{P}}, \mathrm{V} \in \sigma_{\mathrm{POOP}}\right\}$.

Theorem 2.8 ([12]). Let $\mathrm{P}$ be a doubly continuous poset. Then $\bar{\sigma}_{\mathrm{P}}=\mathcal{T}_{\mathrm{P}}$.

Depending on the introduction and discussion of B-topology on posets [12], we are now in the position to clarify the order-theoretical condition for posets, under which the $\mathrm{O}_{1}$-convergence is topological.

Definition 2.9. The $\mathrm{O}_{1}$-convergence in a poset $\mathrm{P}$ is said to be topological if there exists a topology $\mathcal{T}$ on $\mathrm{P}$ such that

$$
\left(x_{i}\right)_{i \in \mathrm{I}} \stackrel{\mathcal{T}}{\rightarrow} x \in \mathrm{P} \Longleftrightarrow\left(x_{i}\right)_{i \in \mathrm{I}} \stackrel{\mathrm{O}_{1}}{\longrightarrow} x .
$$

Definition 2.10. An $\mathrm{S}^{*}$-doubly continuous poset $\mathrm{P}$ is said to be consistent with respect to the $\mathrm{O}_{1}$-convergence (for short, B-consistent) if it satisfies the following condition for any net $\left(x_{i}\right)_{i \in I}$ in $P$ and any $x \in P$ :

$$
x_{i} \in \uparrow y \cap \downarrow z \text { eventually for any } y \in \Downarrow_{S} x \text { and } z \in \uparrow_{s} x \Rightarrow\left(x_{i}\right)_{i \in I} \stackrel{\mathrm{O}_{1}}{\longrightarrow} x .
$$

Lemma 2.11. If $\mathrm{P}$ is a B-consistent $\mathrm{S}^{*}$-doubly continuous poset, then the $\mathrm{O}_{1}$-convergence in $\mathrm{P}$ is topological. 
Proof. By Remark 2.3 (2) and Proposition 2.5, it is not difficult to show a net

$$
\left(x_{i}\right)_{i \in I} \stackrel{\mathrm{O}_{1}}{\longrightarrow} x \in P \Longrightarrow\left(x_{i}\right)_{i \in I} \stackrel{\mathcal{T}_{P}}{\longrightarrow} x .
$$

To prove the theorem, it suffices to show a net

$$
\left(x_{i}\right)_{i \in I} \stackrel{\mathcal{T}_{P}}{\longrightarrow} x \in P \Longrightarrow\left(x_{i}\right)_{i \in I} \stackrel{\mathrm{O}_{1}}{\longrightarrow} x .
$$

Now we suppose the net $\left(x_{i}\right)_{i \in I} \stackrel{\mathcal{T}_{P}}{\longrightarrow} x$. For any $y \in \Downarrow_{S} x$ and $z \in \uparrow_{s} x$, since $P$ is an $S^{*}$-doubly continuous poset, there exist $y_{0} \in \Downarrow_{s} x$ and $z_{0} \in \uparrow_{s} x$ such that $\uparrow_{0} \cap \downarrow_{0} \subseteq \Uparrow s y \cap \downarrow_{s} z$. According to Theorem 2.6, we have that $x_{i} \in \Uparrow s y_{0} \cap \downarrow s z_{0}$ eventually. Thus $x_{i} \in \Uparrow s y_{0} \cap \downarrow_{s} z_{0} \subseteq \uparrow y_{0} \cap \downarrow z_{0} \subseteq \Uparrow s y \cap \downarrow_{s} z \subseteq \uparrow y \cap \downarrow z$ holds eventually. It follows from the assumption of $\mathrm{P}$ satisfying condition $(\star)$ that the net $\left(x_{i}\right)_{i \in \mathrm{I}} \stackrel{\mathrm{O}_{1}}{\longrightarrow} x$.

Conversely, we have the following Lemma.

Lemma 2.12. If the $\mathrm{O}_{1}$-convergence in a poset $\mathrm{P}$ is topological, then $\mathrm{P}$ is a B-consistent $\mathrm{S}^{*}$-doubly continuous poset.

The proof of Lemma 2.12 is divided into the following three steps (Facts 2.13, 2.14, and 2.15).

Fact 2.13. If the $\mathrm{O}_{1}$-convergence in a poset $\mathrm{P}$ is topological, then $\mathrm{P}$ is an $\mathrm{S}$-doubly continuous poset.

Proof. Suppose that the $\mathrm{O}_{1}$-convergence in $\mathrm{P}$ is topological. Then there exists a topology $\mathcal{T}$ on $\mathrm{P}$ such that a net

$$
\left(x_{i}\right)_{i \in I} \stackrel{\mathrm{O}_{1}}{\rightarrow} x \Longleftrightarrow\left(x_{i}\right)_{i \in I} \stackrel{\mathcal{T}}{\rightarrow} x
$$

for every $x \in P$. Let $\mathcal{D}=\left\{(w, W) \in\left(\cup \mathcal{N}_{x}\right) \times \mathcal{N}_{x}: \mathcal{W} \in W\right\}$, where $\mathcal{N}_{x}=\{W \in \mathcal{T}: x \in W\}$. Define the order $\leqslant$ on $\mathcal{D}$ by

$$
\left(\forall\left(w_{1}, W_{1}\right),\left(w_{2}, W_{2}\right) \in \mathcal{D}\right)\left(w_{1}, W_{1}\right) \leqslant\left(w_{2}, W_{2}\right) \Longleftrightarrow W_{2} \subseteq W_{1} .
$$

It can be verified straightforwardly that $\leqslant$ is a preorder and $\mathcal{D}$ is directed. Let $x_{(w, W)}=w$ for every $(w, W) \in \mathcal{D}$. Then for any $V \in \mathcal{N}_{x}$, we have that $x_{(w, W)}=w \in W \subseteq V$ for every $(w, W) \geqslant(x, V)$. This means the net $\left(x_{(w, W)}\right)_{(w, W) \in \mathcal{D}} \stackrel{\mathcal{T}}{\rightarrow} x$. Hence $\left(x_{(w, W)}\right)_{(w, W) \in \mathcal{D}} \stackrel{\mathrm{O}_{1}}{\rightarrow} x$. And thus, there exist an increasing net $\left(u_{(w, W)}\right)_{(w, W) \in \mathcal{D}}$ and a decreasing net $\left(v_{(w, W)}\right)_{(w, W) \in \mathcal{D}}$ satisfying the conditions (O2) and (O3) in Definition 2.1.

Let $\mathrm{D}=\left\{\mathfrak{u}_{(w, W)}:(w, W) \in \mathcal{D}\right\}$ and $\mathrm{F}=\left\{v_{(w, W)}:(w, W) \in \mathcal{D}\right\}$. Then by Definition 2.1 and Remark $2.3(2)$, $\mathrm{D}$ is directed, $\mathrm{F}$ is filtered, and $\sup \mathrm{D}=\mathrm{x}=\mathrm{inf} \mathrm{F}$. For every $\mathrm{d}=\mathfrak{u}_{\left(w_{1}, W_{1}\right)} \in \mathrm{D}$ and $e=v_{\left(w_{2}, W_{2}\right)} \in \mathrm{F}$, as $\left(w, W_{1} \cap\right.$ $\left.W_{2}\right) \geqslant\left(w_{1}, W_{1}\right),\left(w_{2}, W_{2}\right)$ for every $w \in W_{1} \cap W_{2}$, we can conclude that $d=\mathfrak{u}_{\left(w_{1}, W_{1}\right)} \leqslant \mathfrak{u}_{\left(w, W_{1} \cap W_{2}\right)} \leqslant$ $x_{\left(w, W_{1} \cap W_{2}\right)}=w \leqslant v_{\left(w, W_{1} \cap W_{2}\right)} \leqslant v_{\left(w_{2}, W_{2}\right)}=e$ for each $w \in W_{1} \cap W_{2}$, which implies $W_{1} \cap W_{2} \subseteq \uparrow d \cap \downarrow e$. Let $D^{\prime}$ be a directed subset of $P$ with sup $D^{\prime}=x$. Consider the net $\left(x_{d^{\prime}}\right) d^{\prime} \in D^{\prime}$ defined by $x_{d^{\prime}}=d^{\prime}$ for all $d^{\prime} \in D^{\prime}$. By Remark 2.3 (4), the net $\left(x_{d^{\prime}}\right)_{d^{\prime} \in D^{\prime}} \stackrel{O_{1}}{\longrightarrow} x$. This implies the net $\left(x_{d^{\prime}}\right)_{d^{\prime} \in D^{\prime}} \stackrel{\mathcal{T}}{\rightarrow} x$. Thus, there exists $d_{0}^{\prime} \in D^{\prime}$ such that $x_{\mathrm{d}^{\prime}}=\mathrm{d}^{\prime} \in \mathrm{W}_{1} \cap \mathrm{W}_{2} \subseteq \uparrow \mathrm{d} \cap \downarrow$ e for every $\mathrm{d}^{\prime} \geqslant \mathrm{d}_{0}^{\prime}$. In particular, we have $\mathrm{x}_{\mathrm{d}_{0}^{\prime}}=\mathrm{d}_{0}^{\prime} \in \uparrow \mathrm{d} \cap \downarrow e$, and thus $\mathrm{d} \ll s \mathrm{~s}$. Because $d$ is arbitrarily taken from $D$, we have $D \subseteq \Downarrow_{s} x$ and $\sup D=\sup \Downarrow_{s} x=x$. For $y_{1}, y_{2} \in \Downarrow_{s} x$, since $D$ is directed and $\sup D=x$, by the definition of $\ll s$, there exist $d_{1}, d_{2} \in D$ such that $y_{1} \leqslant d_{1}$ and $y_{2} \leqslant d_{2}$. Hence there exists $d_{0} \in D \subseteq \Downarrow_{S} x$ such that $y_{1} \leqslant d_{1} \leqslant d_{0}$ and $y_{2} \leqslant d_{2} \leqslant d_{0}$. Therefore, the set $\Downarrow_{S} x$ is directed. It can be similarly proved that the set $\uparrow s_{s} \chi$ is filtered and inf $\uparrow s x=\chi$. Therefore, $P$ is $S$-doubly continuous.

Fact 2.14. If the $\mathrm{O}_{1}$-convergence in a poset $\mathrm{P}$ is topological, then $\mathrm{P}$ is an $\mathrm{S}^{*}$-doubly continuous poset.

Proof. For any $y \in \Downarrow_{s} x$ and $z \in \uparrow_{s} x$, it follows from the definitions of $\ll_{s}$ and $\triangleright_{s}$ that there exist $d_{y}=u_{\left(w_{3}, W_{3}\right)} \in$ $\mathrm{D}$ and $e_{z}=v_{\left(w_{4}, W_{4}\right)} \in \mathrm{F}$ such that $\mathrm{y} \leqslant \mathrm{d}_{y}$ and $e_{z} \leqslant z$. By the discussion in Fact 2.13, we have $W_{3} \cap W_{4} \subseteq$ $\uparrow d_{y} \cap \downarrow e_{z} \subseteq \uparrow y \cap \downarrow z . I_{x}=\left\{\uparrow a \cap \downarrow b: a \in \Downarrow_{s} x \& b \in \uparrow_{s} x\right\}$ and $I_{0}=\left\{(k, K) \in\left(\cup I_{x}\right) \times I_{x}: k \in K\right\}$. And define the order $\prec$ on $\mathrm{I}_{0}$ by

$$
\left(\forall\left(\mathrm{k}_{1}, \mathrm{~K}_{1}\right),\left(\mathrm{k}_{2}, \mathrm{~K}_{2}\right) \in \mathrm{I}_{0}\right)\left(\mathrm{k}_{1}, \mathrm{~K}_{1}\right) \prec\left(\mathrm{k}_{2}, \mathrm{~K}_{2}\right) \Longleftrightarrow \mathrm{K}_{2} \subseteq \mathrm{K}_{1} .
$$


One can easily check that $\prec$ is a directed preoder. Let $x_{(k, K)}=k, u_{(k, K)}=a$ and $v_{(k, K)}=b$ for every $(k, K)=$ $(k, \uparrow a \cap \downarrow b) \in I_{0}$. Then the nets $\left(x_{(k, K)}\right)_{(k, K) \in I_{0}},\left(u_{(k, K)}\right)_{(k, K) \in I_{0}}$ increasing) and $\left(v_{(k, K)}\right)_{(k, K) \in I_{0}}($ decreasing) satisfy the conditions $(\mathrm{O} 2)$ and $(\mathrm{O} 3)$ in Definition 2.1, and hence the net $\left(\mathrm{x}_{(\mathrm{k}, \mathrm{K})}\right)_{(\mathrm{k}, \mathrm{K}) \in \mathrm{I}_{0}} \stackrel{\mathrm{O}_{1}}{\longrightarrow} \mathrm{x}$. Thus, the net $\left(\mathrm{x}_{(\mathrm{k}, \mathrm{K})}\right)_{(\mathrm{k}, \mathrm{K}) \in \mathrm{I}_{0}} \stackrel{\mathcal{T}}{\rightarrow} \mathrm{x}$. This means that there exists $\left(\mathrm{k}_{0}, \mathrm{~K}_{0}\right)=\left(\mathrm{k}_{0}, \uparrow \mathrm{y}_{0} \cap \downarrow z_{0}\right) \in \mathrm{I}_{0}$, where $\mathrm{y}_{0} \in \Downarrow_{\mathrm{s}} x$ and $z_{0} \in \uparrow \uparrow_{\mathrm{s}} x$, such that $x_{(k, K)}=k \in W_{3} \cap W_{4} \subseteq \uparrow d_{y} \cap \downarrow e_{z} \subseteq \uparrow y \cap \downarrow z$ for all $(k, K) \succ\left(k_{0}, K_{0}\right)$. In particular, it holds that $\left(k, K_{0}\right) \succ\left(k_{0}, K_{0}\right)$ for all $k \in K_{0}$, which implies $x_{\left(k, K_{0}\right)}=k \in W_{3} \cap W_{4} \subseteq \uparrow d_{y} \cap \downarrow e_{z} \subseteq \uparrow y \cap \downarrow z$ for all $k \in K_{0}$. Thus, we conclude that $\mathrm{K}_{0}=\uparrow \mathrm{y}_{0} \cap \downarrow z_{0} \subseteq \mathrm{W}_{3} \cap \mathrm{W}_{4} \subseteq \uparrow \mathrm{d}_{\mathrm{y}} \cap \downarrow e_{z} \subseteq \uparrow \mathrm{y} \cap \downarrow z$.

We finally show $\uparrow y_{0} \cap \downarrow z_{0} \subseteq \Uparrow s y \cap \downarrow_{s} z$. Suppose that $D^{\prime \prime}$ is a directed subset of $P$ with sup $D^{\prime \prime}=r \in$ $\uparrow y_{0} \cap \downarrow z_{0}$. Then by Remark 2.3 (4), the net $\left(x_{\mathrm{d}^{\prime \prime}}\right)_{\mathrm{d}^{\prime \prime} \in \mathrm{D}^{\prime \prime}}$ defined by $\mathrm{x}_{\mathrm{d}^{\prime \prime}}=\mathrm{d}^{\prime \prime}$ for all $\mathrm{d}^{\prime \prime} \in \mathrm{D}^{\prime \prime} \mathrm{O}_{1}$-converges to $\mathrm{r}$, and thus the net $\left(x_{\mathrm{d}^{\prime \prime}}\right)_{\mathrm{d}^{\prime \prime} \in \mathrm{D}^{\prime \prime}} \stackrel{\mathcal{T}}{\rightarrow} \mathrm{r}$. It follows that there exists $\mathrm{d}_{0}^{\prime \prime} \in \mathrm{D}^{\prime \prime}$ such that $\mathrm{x}_{\mathrm{d}_{0}^{\prime \prime}}=\mathrm{d}_{0}^{\prime \prime} \in \mathrm{W}_{3} \cap \mathrm{W}_{4} \subseteq \uparrow \mathrm{y} \cap \downarrow z$, which implies $y \ll s r$. It is analogous to prove $z \triangleright{ }_{s} r$. Hence, we have $\uparrow y_{0} \cap \downarrow z_{0} \subseteq \Uparrow s y \cap \downarrow_{s} z$. Therefore P is an $S^{*}$-doubly continuous poset.

Fact 2.15. If the $\mathrm{O}_{1}$-convergence in a poset $\mathrm{P}$ is topological, then $\mathrm{P}$ satisfies condition $(\star)$.

Proof. Let $\left(x_{j}\right)_{j \in J}$ be a net in $P$ with $x_{j} \in \uparrow y \cap \downarrow z$ eventually for any $y \in \Downarrow_{S} x$ and $z \in \uparrow_{s} x$. By what has been shown in Fact 2.14, the net $\left(x_{(k, K)}\right)_{(k, K) \in I_{0}} \stackrel{\mathcal{T}}{\rightarrow} x$. This means that for every $W \in \mathcal{N}_{x}$ there exists $\left(k_{1}, K_{1}\right)=\left(k_{1}, \uparrow y_{1} \cap\right.$ $\left.\downarrow z_{1}\right) \in \mathrm{I}_{0}$, where $\mathrm{y}_{1} \in \Downarrow_{S} x$ and $z_{1} \in \uparrow_{\mathrm{s}} x$, such that $\mathrm{x}_{(\mathrm{k}, \mathrm{K})}=\mathrm{k} \in \mathrm{W}$ for all $(\mathrm{k}, \mathrm{K}) \succ\left(\mathrm{k}_{1}, \mathrm{~K}_{1}\right)$. In particular, $\left(k, K_{1}\right) \succ\left(k_{1}, K_{1}\right)$ for all $k \in K_{1}$, i.e., $x_{\left(k, K_{1}\right)}=k \in W$ for all $k \in K_{1}$, which implies $K_{1}=\uparrow y_{1} \cap \downarrow z_{1} \subseteq W$. By the assumption that $x_{j} \in \uparrow y_{1} \cap \downarrow z_{1} \subseteq W$ eventually, it follows that the net $\left(x_{j}\right)_{j \in J} \stackrel{\mathcal{I}}{\rightarrow} x$. Therefore, the net $\left(x_{j}\right)_{j \in J} \stackrel{O_{1}}{\longrightarrow} x$. This shows $P$ satisfies condition $(\star)$.

The combination of Lemma 2.11 and Lemma 2.12 gives the main result of this paper.

Theorem 2.16. For a poset $\mathrm{P}$ the following statements are equivalent:

(1) The $\mathrm{O}_{1}$-convergence in $\mathrm{P}$ is topological.

(2) $\mathrm{P}$ is a B-consistent $\mathrm{S}^{*}$-doubly continuous poset.

(3) For any net $\left(x_{i}\right)_{i \in I}$ in $\mathrm{P},\left(x_{i}\right)_{i \in \mathrm{I}} \stackrel{\mathrm{O}_{1}}{\longrightarrow} x \in \mathrm{P} \Longleftrightarrow\left(x_{i}\right)_{i \in \mathrm{I}} \stackrel{\mathcal{T}_{\mathrm{P}}}{\longrightarrow} x$.

Proof.

$(1) \Rightarrow(2)$ : By Lemma 2.12 .

$(2) \Rightarrow(3)$ : By the proof of Lemma 2.11 .

(3) $\Rightarrow(1)$ : It is straightforward.

Corollary 2.17. Let $\mathrm{P}$ be a B-consistent doubly continuous poset. Then

(1) The $\mathrm{O}_{1}$-convergence in $\mathrm{P}$ is topological.

(2) For any net $\left(x_{i}\right)_{i \in I}$ in $\mathrm{P},\left(x_{i}\right)_{i \in \mathrm{I}} \stackrel{\mathrm{O}_{1}}{\longrightarrow} x \in \mathrm{P} \Longleftrightarrow\left(x_{i}\right)_{i \in \mathrm{I}} \stackrel{\mathcal{T}_{P}}{\longrightarrow} x$.

(3) For any net $\left(x_{i}\right)_{i \in I}$ in $\mathrm{P},\left(x_{i}\right)_{i \in I} \stackrel{\mathrm{O}_{1}}{\longrightarrow} x \in \mathrm{P} \Longleftrightarrow\left(x_{i}\right)_{i \in \mathrm{I}} \stackrel{\bar{\sigma}_{\mathrm{P}}}{\longrightarrow} x$.

Proof.

(1): By Proposition 1.9 and Theorem 2.16.

(2): By Proposition 1.9 and Theorem 2.16.

(3): By Theorems 2.8 and 2.16.

\section{Special B-consistent $S^{*}$-doubly continuous posets}

In this section, we will discuss several special B-consistent $S^{*}$-doubly continuous posets. We first give a basic property of B-consistent $\mathrm{S}^{*}$-doubly continuous posets. 
Proposition 3.1. Every B-consistent $\mathrm{S}^{*}$-doubly continuous poset is bounded.

Proof. Suppose that $\mathrm{P}$ is a B-consistent $\mathrm{S}^{*}$-doubly continuous poset. Let $\mathrm{I}=\mathrm{P} \cup\{0,1\}$ and define the order $\leqslant$ on I as follows: $0 \leqslant i \leqslant 1$ for every $i \in I$. Then $I$ is directed since 1 is the largest element in I. For every $x \in P$, let $x_{0}=x_{1}=x$ and $x_{i}=i$ for all $i \in P$. Since $x_{1}=x \in \uparrow y \cap \downarrow z$ for every $y \in \Downarrow_{S} x$ and $z \in \uparrow_{S} x$, by condition $(\star)$, the net $\left(x_{i}\right)_{i \in I} \stackrel{\mathrm{O}_{1}}{\longrightarrow} x$. This means that there exist an increasing net $\left(u_{i}\right)_{i \in I}$ and a decreasing net $\left(v_{i}\right)_{i \in I}$ satisfying the conditions (O2) and (O3) in Definition 2.1, which implies that $u_{0} \leqslant x_{i}=i \leqslant v_{0}$ for all $i \in P$. Thus $u_{0}$ is the least element in $\mathrm{P}$ and $v_{0}$ is the largest element in $\mathrm{P}$. Therefore, $\mathrm{P}$ is bounded.

A ordinal number $\lambda$, from the order-theoretical point of view, is indeed a linearly ordered set of which every nonempty subset has the least element. It is easy to see that if a subset $\lambda_{0}$ of $\lambda$ has an upper bound in $\lambda$, then sup $\lambda_{0}=\min U\left(\lambda_{0}\right)$, where $U\left(\lambda_{0}\right)$ is the set of all upper bound of $\lambda_{0}$ in $\lambda$. One can refer to [2] for a more detailed discussion about ordinal numbers.

Let $P$ and $Q$ be two posets. A mapping $f: P \rightarrow Q$ is an order embedding if for any $x, y \in P, f(x) \leqslant Q f(y)$ in $Q$ if and only if $x \leqslant p y$ in $P$. Similarly, a mapping $g: P \rightarrow Q$ is a dual order embedding if for any $x, y \in P$, $g(x) \leqslant Q g(y)$ in $Q$ if and only if $x \geqslant_{P} y$ in $P$. We should note that both $f$ and $g$ are injective but need not to be bijective.

Let $\mathrm{P}$ and $\mathrm{Q}$ be two posets and $\mathrm{Q}_{1}, \mathrm{Q}_{2} \subseteq \mathrm{Q}$. An order embedding $f: \mathrm{P} \rightarrow \mathrm{Q}$ is said to be cofinal in $Q_{1}$ if $f(P) \subseteq Q_{1}$, and for any $q_{1} \in Q_{1}$ there exists $p_{1} \in P$ such that $f\left(p_{1}\right) \geqslant Q_{1} q_{1}$. Similarly, a dual order embedding $g: P \rightarrow Q$ is said to be dually cofinal in $Q_{2}$ if $g(P) \subseteq Q_{2}$, and for any $q_{2} \in Q_{2}$ there exists $\mathrm{p}_{2} \in \mathrm{P}$ such that $\mathrm{g}\left(\mathrm{p}_{2}\right) \leqslant \mathrm{Q} \mathrm{q}_{2}$.

Definition 3.2. An $S^{*}$-doubly continuous poset $P$ is called locally ordinal embedded if for every $x \in P$, there exist ordinal numbers $\lambda_{x}, \mu_{x}$, an order embedding $f_{x}: \lambda_{x} \rightarrow P$ and a dual order embedding $g_{x}: \mu_{x} \rightarrow P$ such that $f_{x}$ is cofinal in $\Downarrow_{S} x$ and $g_{x}$ is dually cofinal in $\uparrow_{s} x$.

Proposition 3.3. Let $\mathrm{P}$ be an $\mathrm{S}^{*}$-doubly continuous poset. If $\mathrm{P}$ is locally ordinal embedded and bounded, then it is B-consistent.

Proof. Suppose that an $S^{*}$-doubly continuous poset $\mathrm{P}$ is locally ordinal embedded and bounded. Then for every $x \in P$, there exist ordinal numbers $\lambda_{x}, \mu_{x}$, an order embedding $f_{x}: \lambda_{x} \rightarrow P$ and a dual order embedding $g_{x}: \mu_{x} \rightarrow P$ such that $f_{x}$ is cofinal in $\Downarrow_{s} x$ and $g_{x}$ is dually cofinal in $\uparrow_{s} x$. Let $\left(x_{i}\right)_{i \in I}$ be a net in $P$ with that $x_{i} \in \uparrow y \cap \downarrow z$ eventually for every $y \in \Downarrow_{s} x$ and $z \in \uparrow_{s} x$. For every $i \in I$, we define

$$
u_{i}= \begin{cases}x, & \left\{\lambda \in \lambda_{x}:(\forall j \geqslant i) x_{j} \geqslant f_{x}(\lambda)\right\}=\lambda, \\ f_{x}\left(\sup \left\{\lambda \in \lambda_{x}:(\forall j \geqslant i) x_{j} \geqslant f_{x}(\lambda)\right\}\right), & \emptyset \neq\left\{\lambda \in \lambda_{x}:(\forall j \geqslant i) x_{j} \geqslant f_{x}(\lambda)\right\} \varsubsetneqq \lambda_{x}, \\ \perp, & \left\{\lambda \in \lambda_{x}:(\forall j \geqslant i) x_{j} \geqslant f_{x}(\lambda)\right\}=\emptyset,\end{cases}
$$

and

$$
v_{i}= \begin{cases}x, & \left\{\mu \in \mu_{x}:(\forall j \geqslant i) x_{j} \leqslant g_{x}(\mu)\right\}=\mu_{x}, \\ g_{x}\left(\sup \left\{\mu \in \mu_{x}:(\forall j \geqslant i) x_{j} \leqslant g_{x}(\mu)\right\}\right), & \emptyset \neq\left\{\mu \in \mu_{x}:(\forall j \geqslant i) x_{j} \leqslant g_{x}(\mu)\right\} \mp \mu_{x}, \\ \top, & \left\{\mu \in \mu_{x}:(\forall j \geqslant i) x_{j} \leqslant g_{x}(\mu)\right\}=\emptyset,\end{cases}
$$

where $\perp$ and $T$ are the least and largest elements of $P$, respectively .

It is tedious but straightforward to check that the nets $\left(u_{i}\right)_{i \in I},\left(v_{i}\right)_{i \in I}$ are respectively increasing and decreasing, and satisfy the conditions $(\mathrm{O} 2)$ and $(\mathrm{O} 3)$ in Definition 2.1. Thus the net $\left(x_{i}\right)_{i \in I} \stackrel{\mathrm{O}_{1}}{\longrightarrow} x$. By Definition 2.10, P is B-consistent.

Definition 3.4. We say that an $S^{*}$-doubly continuous poset $P$ is locally countable if $\left|\Downarrow_{S} \chi\right|,\left|\uparrow \uparrow_{s} x\right| \leqslant \omega_{0}$ for every $x \in P$, where $\left|\Downarrow_{S} x\right|$ and $\left|\uparrow_{s} x\right|$ denote the cardinalities of the sets $\Downarrow_{S} x$ and $\uparrow_{s} x$, respectively, and $\omega_{0}$ is the first infinite cardinal number.

Proposition 3.5. Let $\mathrm{P}$ be an $\mathrm{S}^{*}$-doubly continuous poset. If $\mathrm{P}$ is locally countable, then $\mathrm{P}$ is locally ordinal embedded. 
Proof. For every $x \in \mathrm{P}$, we consider the following two cases:

(Case 1.) If $x \in \Downarrow_{S} x$, let $\lambda_{x}=1$ and define $f_{x}: 1 \rightarrow P$ by $f_{x}(0)=x$. Now we can easily see that $f_{x}: 1 \rightarrow P$ is an order embedding and is cofinal in $\Downarrow_{S} x$.

(Case 2.) If $x \notin \Downarrow_{S} x$, then $\Downarrow_{S} x$ is directed and $\left|\Downarrow_{S} x\right|=\omega_{0}$ since $P$ is $S^{*}$-doubly continuous and locally countable. Without loss of generality, we assume $\Downarrow_{s} x=\left\{y_{0}, y_{1}, y_{2}, y_{3}, \ldots\right\}$. Let $\lambda_{x}=\omega_{0}$ and define $f_{x}: \omega_{0} \rightarrow P$ by inductive approach with respect to $\omega_{0}$ :

(Step 1.) Let $f_{x}(0)=y_{0}$.

(Step 2.) Suppose that all of $f_{x}(0), f_{x}(1), f_{x}(2), \ldots, f_{x}(n)$ have been defined. Then we can take a $y^{n+1} \in \Downarrow_{s} x$ such that $y^{n+1}>y_{i}$ and $y^{n+1}>f_{x}(i)$ for all $i \leqslant n$. Now let $f_{x}(n+1)=y^{n+1}$.

It is clear that $f_{x}: \omega_{0} \rightarrow P$ is an order embedding which is cofinal in $\Downarrow_{S} x$.

In sum, we have proved that there exist an ordinal numbers $\lambda_{x}$ and an order embedding $f_{x}: \lambda_{x} \rightarrow P$ such that $f_{x}$ is cofinal in $\Downarrow_{S} x$. It can be similarly showed that there exist an ordinal numbers $\mu_{x}$ and a dual order embedding $g_{x}: \mu_{x} \rightarrow P$ such that $g_{x}$ is dually cofinal in $\uparrow_{s} x$. Hence $P$ is locally ordinal embedded.

Definition 3.6. An $S^{*}$-doubly continuous poset $P$ is said to be weakly locally countable if for every $x \in P$ there always exist a countable directed subset $D_{x}$ of $\Downarrow_{s} \chi$ and a countable filtered subset $F_{\chi}$ of $\uparrow_{s} \chi$ such that $\sup D_{x}=\chi=\inf F_{\chi}$.

Proposition 3.7. Let $\mathrm{P}$ be an $\mathrm{S}^{*}$-doubly continuous poset. If $\mathrm{P}$ is weakly locally countable, then $\mathrm{P}$ is locally ordinal embedded.

Proof. Suppose that an $\mathrm{S}^{*}$-doubly continuous poset $\mathrm{P}$ is weakly locally countable. Then for every $x \in \mathrm{P}$, there exist a countable directed subset $D_{x}$ of $\Downarrow_{S} x$ and a countable filtered subset $F_{x}$ of $\uparrow_{s} x$ such that $\sup D_{x}=x=\inf F_{x}$. By the technique of induction used in the proof of Proposition 3.5, we can get ordinal numbers $\lambda_{x}, \mu_{x}$, an order embedding $f_{x}: \lambda_{x} \rightarrow P$ and a dual order embedding $g_{x}: \mu_{x} \rightarrow P$ such that $f_{x}$ is cofinal in $D_{x}$ and $g_{x}$ is dually cofinal in $F_{x}$. Furthermore, One can easily check by the definitions of $\ll_{s}$ and $\triangleright_{S}$ that $f_{x}$ is also cofinal in $\Downarrow_{s} x$ and $g_{x}$ is dually cofinal in $\uparrow_{s} x$. Hence $P$ is locally ordinal embedded.

Theorem 3.8. If $\mathrm{P}$ is an $\mathrm{S}^{*}$-doubly continuous, locally ordinal embedded and bounded poset, then the $\mathrm{O}_{1}$-convergence in $\mathrm{P}$ is topological.

Proof. Straightforwardly from Theorem 2.16 and Proposition 3.3.

Corollary 3.9. Let $\mathrm{P}$ be a poset. Then

(1) If $\mathrm{P}$ is $\mathrm{S}^{*}$-doubly continuous, bounded and locally countable, then the $\mathrm{O}_{1}$-convergence in $\mathrm{P}$ is topological.

(2) If $\mathrm{P}$ is a countable poset, i.e., $|\mathrm{P}| \leqslant \omega_{0}$, then the $\mathrm{O}_{1}$-convergence in $\mathrm{P}$ is topological if and only if $\mathrm{P}$ is $\mathrm{S}^{*}$-doubly continuous and bounded.

(3) If $\mathrm{P}$ is a finite poset, i.e., $|\mathrm{P}|<\omega_{0}$, then the $\mathrm{O}_{1}$-convergence in $\mathrm{P}$ is topological if and only if $\mathrm{P}$ is bounded.

Proof.

(1): Straightforwardly follows from Proposition 3.5 and Theorem 3.8.

(2): Let $\mathrm{P}$ be a countable poset. If the $\mathrm{O}_{1}$-convergence in $\mathrm{P}$ is topological. Then, by Theorem $2.16, \mathrm{P}$ is a $\mathrm{B}$ consistent $S^{*}$-doubly continuous poset. It follows from Proposition 3.1 that $P$ is bounded. Conversely, suppose that $P$ is $\mathrm{S}^{*}$-doubly continuous and bounded. Since $\mathrm{P}$ is a countable poset, it is locally countable. By Proposition 3.5, $\mathrm{P}$ is locally ordinal embedded. Thus we can conclude by Theorem 3.8 that the $\mathrm{O}_{1}$-convergence in $\mathrm{P}$ is topological.

(3): It is straightforwardly from (2) if we notice the fact that every finite poset is a doubly continuous poset and hence an $\mathrm{S}^{*}$-doubly continuous poset.

Definition 3.10. An $S^{*}$-doubly continuous poset $P$ is said to be locally complete if for any $x \in P, A \subseteq \Downarrow_{S} x$ and $\mathrm{B} \subseteq \uparrow_{s} \mathrm{x}$, both of $\sup \mathrm{A}$ and inf $\mathrm{B}$ in $\mathrm{P}$ exist. 
Proposition 3.11. If $\mathrm{P}$ be an $\mathrm{S}^{*}$-doubly continuous, locally complete and bounded poset, then $\mathrm{P}$ is B-consistent.

Proof. Suppose $\mathrm{P}$ is an $\mathrm{S}^{*}$-doubly continuous, locally complete and bounded poset. Let $\left(x_{i}\right)_{i \in \mathrm{I}}$ be a net in $\mathrm{P}$ with that $x_{i} \in \uparrow y \cap \downarrow z$ eventually for every $y \in \Downarrow_{s} x$ and $z \in \uparrow_{s} x$. For every $i \in I$ we define

$$
u_{i}= \begin{cases}\sup \left\{y^{\prime} \in \Downarrow_{S} x:(\forall j \geqslant i) x_{j} \geqslant y^{\prime}\right\}, & \left\{y^{\prime} \in \Downarrow_{S} x:(\forall j \geqslant i) x_{j} \geqslant y^{\prime}\right\} \neq \emptyset, \\ \perp, & \left\{y^{\prime} \in \Downarrow_{S} x:(\forall j \geqslant i) x_{j} \geqslant y^{\prime}\right\}=\emptyset,\end{cases}
$$

and dually define

$$
v_{i}= \begin{cases}\inf \left\{z^{\prime} \in \uparrow s_{s} x:(\forall j \geqslant i) x_{j} \leqslant z^{\prime}\right\}, & \left\{z^{\prime} \in \uparrow \uparrow_{s} x:(\forall j \geqslant i) x_{j} \leqslant z^{\prime}\right\} \neq \emptyset, \\ \top, & \left\{z^{\prime} \in \uparrow s x:(\forall j \geqslant i) x_{j} \leqslant z^{\prime}\right\}=\emptyset,\end{cases}
$$

where, $\perp$ and $T$ are the least element and largest element of $P$, respectively.

One can straightforwardly verifies that the increasing net $\left(u_{i}\right)_{i \in I}$ and decreasing net $\left(v_{i}\right)_{i \in I}$ satisfy the conditions $(\mathrm{O} 2)$ and (O3) in Definition 2.1. Hence the net $\left(x_{i}\right)_{i \in I} \stackrel{\mathrm{O}_{1}}{\longrightarrow} x$. Thus, $\mathrm{P}$ is B-consistent.

Theorem 3.12. If $\mathrm{P}$ is an $\mathrm{S}^{*}$-doubly continuous, locally complete and bounded poset, then the $\mathrm{O}_{1}$-convergence in $\mathrm{P}$ is topological.

Proof. Straightforwardly from Theorem 2.16 and Proposition 3.11.

Corollary 3.13. Let $\mathrm{P}$ be a poset.

(1) If $\mathrm{P}$ is a complete lattice, then the $\mathrm{O}_{1}$-convergence in $\mathrm{P}$ is topological if and only if $\mathrm{P}$ is $\mathrm{S}^{*}$-doubly continuous.

(2) If $\mathrm{P}$ is a completely distributive lattice, then the $\mathrm{O}_{1}$-convergence in $\mathrm{P}$ is topological.

(3) If $\mathrm{P}$ is a complete chain, then the $\mathrm{O}_{1}$-convergence in $\mathrm{P}$ is topological.

Proof.

(1) Let $\mathrm{P}$ be a complete lattice. If the $\mathrm{O}_{1}$-convergence in $\mathrm{P}$ is topological, then $\mathrm{P}$ is $\mathrm{S}^{*}$-doubly continuous by Theorem 2.16. Conversely, suppose $P$ is an $S^{*}$-doubly continuous poset. Since $P$ is a complete lattice, it is locally complete and bounded. By Theorem 3.12, the $\mathrm{O}_{1}$-convergence in $\mathrm{P}$ is topological.

(2) Noticing that every completely distributive lattice is doubly continuous, and hence is $\mathrm{S}^{*}$-doubly continuous, by (1), the $\mathrm{O}_{1}$-convergence in $\mathrm{P}$ is topological.

(3) Noticing that every complete chain is completely distributive, by (2), the $\mathrm{O}_{1}$-convergence in $\mathrm{P}$ is topological.

\section{Acknowledgment}

The authors would like to thank the anonymous referee for his/her careful reading and valuable comments which have improved the quality and readability of this paper. And this work is supported by the Natural Science Foundation of Hunan Province of China (Grant No.: 2019JJ50406), and the Significant Research and Development Project of Hunan province (Grant No.: 2016JC2014).

\section{References}

[1] G. Birkhoff, Lattice Theory, American Mathematical Society, (1940). 1, 1, 2.1

[2] M. M. Dai, H. Y. Chen, D. W. Zheng, An introduction to axiomatic set theory (in chinese), Science Press, Beijing, (2011). 3

[3] B. A. Davey, H. A. Priestley, Introduction to Lattices and Order, Cambridge University Press, Cambridge, (2002). 1.5

[4] R. Engelking, General Topology, PWN-Polish Scientific Publishers, Warszawa, (1977). 1

[5] O. Frink, Topology in lattice, Trans. Amer. Math. Soc., 51 (1942), 569-582. 1

[6] G. Grierz, K. H. Hofmann, K. Keimel, J. D. Lawson, M. W. Mislove, D. S. Scott, Continuous Lattices and Domain, Camberidge University Press, Cambridge, (2003). 1.1, 1.2, 1.10 
[7] J. L. Kelly, General Topology, Van Nostrand, New York, (1955). 1

[8] J. C. Mathews, R. F. Anderson, A comparison of two modes of order convergence, Proc. Amer. Math. Soc., 18 (1967), 100-104. 1

[9] E. J. Mcshane, Order-Preserving Maps and Integration Processes, Princeton University Press, Princeton, (1953). 1

[10] V. Olejček, Order Convergence and Order Topology on a Poset, Internat. J. Theoret. Phys., 38 (1999), 557-561. 1

[11] Z. Riečanová, Strongly compactly atomistic orthomodular lattices and modular ortholattices, Tatra Mt. Math. Publ., 15 (1998), 143-153. 1

[12] T. Sun, Q. G. Li, L. K. Guo, Birkhoff's order-convergence in partially ordered sets, Topology Appl., 207 (2016), $156-166$. 1.6, 1.7, 1.8, 1.9, 2, 2.4, 2.5, 2.6, 2.8, 2

[13] K. Y. Wang, B. Zhao, Some further result on order-convergence in posets, Topology Appl., 160 (2013), 82-86. 1

[14] E. S. Wolk, On order-convergence, Proc. Amer. Math. Soc., 12 (1961), 379-384. 1

[15] H. Zhang, A note on continuous partially ordered sets, Semigroup Forum, 47 (1993), 101-104. 1.4

[16] D. S. Zhao, The double Scott topology on a lattice, Chin. Ann. Math. Ser. A, 10 (1989), 187-193. 1.3, 2.7

[17] B. Zhao, K. Y. Wang, Order topology and bi-Scott topology on poset, Acta Math. Sin. (Engl. Ser.), 27 (2011), 2101-2106. 1

[18] B. Zhao, D. S. Zhao, Lim-inf-convergence in partially ordered sets, J. Math. Anal. Appl., 309 (2005), 701-708. 1

[19] Y. H. Zhou, B. Zhao, Order-convergence and Lim-inf $\mathcal{J}_{\mathcal{C}}$-convergence in poset, J. Math. Anal. Appl., 325 (2007), $655-664$. $1.5,3$ 